\title{
NuDEX: A new nuclear $\gamma$-ray cascades generator
}

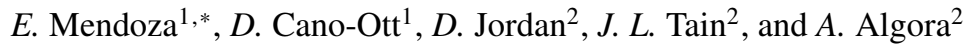 \\ ${ }^{1}$ Centro de Investigaciones Energéticas Medioambientales y Tecnológicas (CIEMAT),Spain \\ ${ }^{2}$ Instituto de Física Corpuscular, CSIC - Universidad de Valencia, Spain
}

\begin{abstract}
Evaluated nuclear data libraries written in ENDF-6 format are used by Monte Carlo codes such as Geant4, MCNP6 or FLUKA for the transport of low energy neutrons (up to $20 \mathrm{MeV}$ ). The format in which the production of $\gamma$-rays after neutron induced reactions is provided do not allow, in general, to generate these $\gamma$-ray cascades in a correlated way. This prevents, among other things, energy conservation event by event, which is crucial in many applications. We have developed a code capable to generate correlated de-excitation $\gamma$-ray cascades using as much information as possible available in the RIPL-3 and ENSDF nuclear structure data libraries, among other useful information. The code follows the same philosophy of the DICEBOX or DEGEN codes. It generates the complete level scheme and branching ratios of the nucleus by using all the information experimentally known (known level scheme and known branching ratios) and completing the missing information with the most reliable statistical models. This code is able to generate automatically cascades for a large variety of nuclei $(\sim 300)$ without requiring a specific input for each particular isotope. The code has been written in $\mathrm{C}++$ language and can be integrated in the Geant 4 simulation toolkit framework.
\end{abstract}

\section{Introduction}

The transport of low energy neutrons (up to $20 \mathrm{MeV}$ ) performed by Monte Carlo codes such as Geant4 [1], MCNP6 [2] or FLUKA [3] usually rely in the information available in evaluated nuclear data libraries, originally written in ENDF-6 format [4] (JEFF-3.3 [5], ENDF/BVIII.0 [6], JENDL-4.0 [7] ...). The information present in these data libraries concerning the $\gamma$-ray emission after nuclear reactions is given in many cases in terms of a list of $n k \gamma$-ray yields $y_{k}\left(E_{n}\right)$, with $k=1,2, \ldots, n k$. Each of these yields represent the average number of $\gamma$-rays of the type $k$ emitted after the corresponding reaction. The yields depend in general on the neutron energy $E_{n}$, and the $\gamma$-ray associated to each $y_{k}\left(E_{n}\right)$ can have either a fixed energy or an energy probability distribution.

From this information it is possible to deduce some quantities such as the total energy of the emitted $\gamma$-ray cascade, the average multiplicity, and the energy distribution of the emitted $\gamma$-rays. However, the information provided does not include correlations between $\gamma$-rays, thus it is not enough to generate the $\gamma$-ray cascades in a correlated way. In particular, this format is not intended to make calculations in which the energy is conserved in each single reaction (but on average) nor contains information on which $\gamma$-rays are emitted in coincidence. The lack of correlations does not affect the accuracy of many Monte Carlo calculations, such as for example the spatial distribution of the energy released by neutron reactions inside a nuclear reactor, dose calculations, or the response of a $\gamma$-ray detector to neutron reactions produced far from the detec- tor. However, difficulties arise when simulating electromagnetic calorimeters, detectors in coincidence, or the response of a detector to $\gamma$-rays from neutron reactions produced near or inside the detector.

There is also the possibility of providing the level scheme and branching ratios in the ENDF-6 format, but in practice this only occurs in a few simple cases, such as in some $\left(\mathrm{n}, \mathrm{n}^{\prime}\right)$ or $\left(\mathrm{n}, \mathrm{p}^{\prime}\right)$ reactions, in which the residual nucleus is left in a well know and low energy excited state.

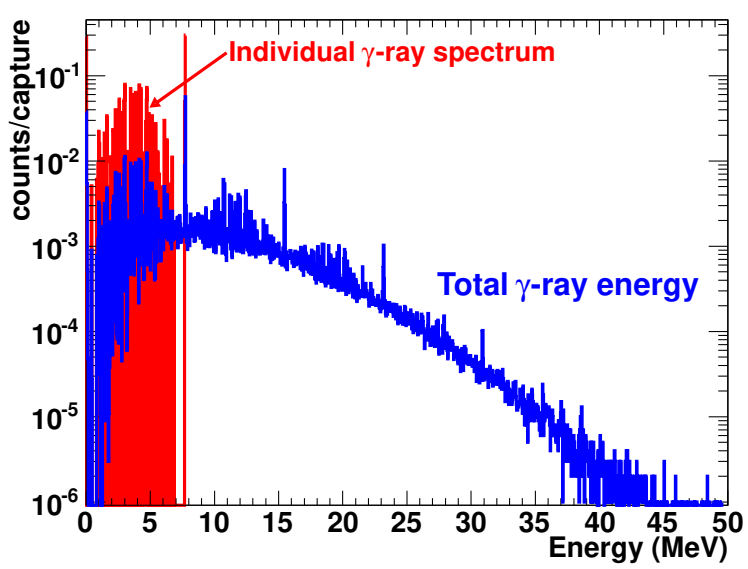

Figure 1. $\gamma$-rays emitted after thermal ${ }^{27} \mathrm{Al}(\mathrm{n}, \gamma)$ reactions in Geant4 using ENDF/B-VII.1. In red, the energy distribution of the individual $\gamma$-rays. In blue, the total cascade energy distribution.

*e-mail: emilio.mendoza@ ciemat.es 
We present an example of the inconsistencies of the current standard cascade generation method in Figure 1. The $\gamma$-rays emitted after ${ }^{27} \mathrm{Al}(\mathrm{n}, \gamma)$ reactions are described by 90 different $y_{k}\left(E_{n}\right)$ yields, i.e. $n k=90$, in the ENDF/BVII.1 evaluated data library. They correspond to one continuum $\gamma$-ray energy distribution and 89 discrete $\gamma$-rays. The procedure followed by Geant 4 to generate each capture cascade is to perform 90 independent random samples, each of them according to a Poisson distribution with mean equal to the corresponding $y_{k}\left(E_{n}\right)$ value, in order to determine how many $\gamma$-rays of each type are produced. Thus, the energy distribution of the emitted $\gamma$-rays is the one provided by ENDF/B-VII.1, and the average total $\gamma$ ray energy released after the capture reactions is $7.73 \mathrm{MeV}$, which corresponds to the neutron separation energy $S_{n}$ of ${ }^{28} \mathrm{Al}$, as expected. Indeed, for low energy neutrons this total energy should be in good approximation $S_{n}\left({ }^{28} \mathrm{Al}\right)$ for every capture reaction. However, the total cascade energy distribution covers the entire range from 0 up to $50 \mathrm{MeV}$. Similar results are obtained with MCNP6.

\section{Description of the code}

With the aim of being able to generate correlated nuclear de-excitation cascades we have developed NuDEX. This code has been written in $\mathrm{C}++$ language and can be integrated in the Geant4 simulation toolkit framework. The methodology of NuDEX for generating the electromagnetic cascades is the same as that of DICEBOX [8] or DEGEN [9]. The code generates the full level scheme and associated branching ratios of the nucleus of interest, considering also the electron conversion processes. For this, $\mathrm{NuDEX}$ has a database taken mainly from ENSDF [10] and RIPL-3 [11], and the missing information is completed with statistical models.

The known part of the level scheme, including branching ratios and internal conversion coefficients (ICC), is obtained from the RIPL-3 database, which takes the data from ENSDF but provides (unlike ENSDF) a unique spin and parity assignment for each level. In addition, the database provides a cut-off energy $\mathrm{E}_{\max }$, up to which a level scheme is supposed to be complete.

The rest of the level scheme is generated randomly according to level density formulas. The distance between consecutive levels are sampled according to a Wigner distribution. For the moment, we have implemented the Back-Shifted Fermi Gas Model (Eq. 48 of [11]) and the Gilbert-Cameron Model (Eqs. 48 and 64 of [11]), as they are defined in the RIPL-3 manual [11] and with the level density parameters provided by the RIPL-3 database. NuDEX can use either of the two models, and new ones can be implemented easily in a future. Known levels above $\mathrm{E}_{\text {max }}$ are inserted in the scheme, whenever possible, after having generated the statistical ones. Each known level above $\mathrm{E}_{\max }$ replaces a statistical one of the same spin and parity, and with similar energy.

Once the full level scheme has been generated, the missing branching ratios are calculated. The branching ratio of the transition from a level $a$ to a level $b, B R_{a \rightarrow b}$, is computed according to [8]:

$$
B R_{a \rightarrow b} \propto \sum_{X, L} \xi_{a \rightarrow b}^{2} E_{\gamma}^{2 L+1} \operatorname{PSF}^{X L}\left(E_{\gamma}, E_{a}\right)
$$

where $X$ is the type of transition (electric or magnetic); $L$ is the multipolarity; $\xi_{a \rightarrow b}$ is a random variable drawn independently from a normal distribution with zero mean and unit variance, which introduce the Porter-Thomas fluctuations; $E_{\gamma}$ is the difference between the energy of the level $a, E_{a}$, and the energy of the level $b, E_{b}$; and $P S F^{X L}$ is the Photon Strength Function (PSF). For the moment, only E1, M1 and E2 transitions are considered.

In addition to the branching ratios from the known part of the level scheme, the NuDEX database contains also lists of primary $\gamma$-rays from thermal neutron capture, together with the observed intensities, taken from ENSDF. These data are particularly important for simulating thermal neutron capture $\gamma$-rays of light nuclei, since otherwise it would not be possible to reproduce the experimental spectra.

By default, PSFs are defined as in the RIPL-3 manual, using the parameters provided by the RIPL-3 database. If these are missing, then they are calculated according to the models and recommendations of the RIPL-3 manual. The PSFs can be also defined by the user, as a linear combination of functions (mainly Lorentzian and Gaussian). At the moment, the Lorentzian functions available are the Standard Lorentzian model (SLO, given by Eq. 135 of [11]), the Generalized Lorentzian model (GLO, Eq. 140 of [11] with $\left.\chi\left(\epsilon_{\gamma}\right)=1\right)$ and the Simplified Modified Lorentzian model (SMLO, Eq. 151 of [11] with the simplified expression in Eq. 159). Finally, the missing ICC parameters are obtained from [12].

NuDEX is able to generate automatically cascades for a large variety of nuclei $(\sim 300)$ without requiring a specific input for each particular isotope. The default options for generating the levels and the transition probabilities can be easily modified, nucleus to nucleus. There is also the possibility of binning the level schemes, making the code go faster and consume less computer memory without significantly affecting the results of the simulation for most practical cases.

\section{Validation and verification}

In order to test the performance of the code we have compared NuDEX capture cascades with the CapGam [13] database and with the DEGEN code. CapGam is provided by the Brookhaven National Laboratory, and consists on a list of $\gamma$-ray energies and intensities from thermal neutron capture for several isotopes. For reasonably well-known light nuclei, the gamma lists are quite complete, and, as the nuclei become heavier, the lists become more and more incomplete.

As an example, we show the comparison between CapGam, NuDEX and other two Geant4 cascade generators in Figure 2 for the case of ${ }^{23} \mathrm{Na}\left(\mathrm{n}_{t h}, \gamma\right)$, but similar results are obtained for other well-known light nuclei. The most precise model for the transport of low energy 

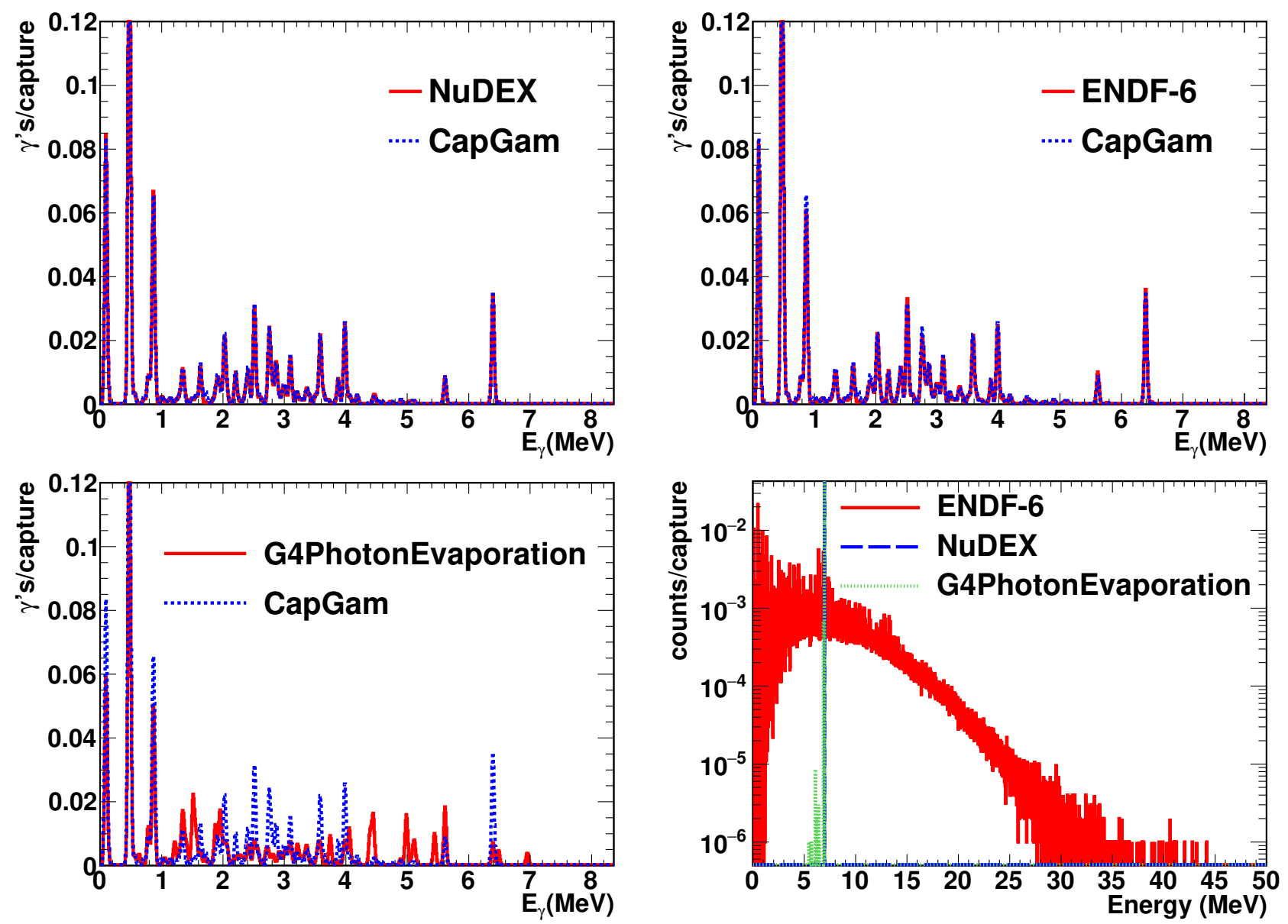

Figure 2. Comparison between CapGam and three different generators for ${ }^{23} \mathrm{Na}\left(\mathrm{n}_{t h}, \gamma\right)$ cascades. Individual $\gamma$-ray intensities from CapGam are compared with NuDEX (top-left), with the standard Geant4 model based on ENDF-6 format data libraries (top-right), and with the Geant4 PhotonEvaporation model (bottom-left). In all these cases the $\gamma$-ray spectra have been broadened by with a resolution $R=50 \mathrm{keV} / E$. On the bottom-right panel, we show the total cascade energy distribution for the three models. There the NuDEX and G4PhotonEvaporation curves practically overlap.

neutrons (up to $20 \mathrm{MeV}$ ) is the so called G4ParticleHP model, which uses the information available in ENDF-6 format data libraries. G4ParticleHP allows also the possibility of deactivating the generation of capture $\gamma$-rays according to ENDF-6 data, and using an alternative model, G4PhotonEvaporation, which generates the cascades in a correlated way, similar to NuDEX but rather simpler. Figure 2 shows that NuDEX reproduces the $\gamma$-ray energy spectrum from CapGam, as does the default-G4ParticleHP (ENDF-6) but not G4PhotonEvaporation, and conserves the energy of the cascade, as does G4PhotonEvaporation and not the default-G4ParticleHP.

For medium and heavy nuclei the cascades from CapGam are not longer complete. For this reason we have compared NuDEX with DEGEN. This comparison was performed with a version of DEGEN in which the level schemes are not binned [14]. For the comparison we used in both codes the same known part of the level scheme, level densities, PSFs ... and we switched off the PorterThomas fluctuations in the branching ratios to reduce the effect of the random number chains in the final results. We have obtained very similar results with both codes in several cases tested, one of them shown in Figure 3.

\section{Results}

In order to show the capabilities of NuDEX we present in Figure 4 an example of the calculation, performed with Geant4, of the response function of a CsI detector to thermal neutrons. We have used the same three models as in the previous section for the generation of the electromagnetic cascades. The two nuclei involved in the simulation are ${ }^{133} \mathrm{Cs}$ and ${ }^{127} \mathrm{I}$, whose capture cascades have a total energy approximately equal to the neutron separation energy $\mathrm{S}_{n}$ of ${ }^{134} \mathrm{Cs}$, which is $6.9 \mathrm{MeV}$, and ${ }^{128} \mathrm{I}$, which is $6.8 \mathrm{MeV}$.

As expected, the default G4ParticleHP model do not conseve the energy of the cascades, leading to unphysical results with deposited energies of up to more than $15 \mathrm{MeV}$. On the contrary, both the G4PhotonEvaporation model and NuDEX produce deposited energy spectra with the maximum in the right place, $6.9 \mathrm{MeV}$. The individual $\gamma$-ray energy spectrum from NuDEX, shown in Figure 5, is very similar to the one obtained when using the information 


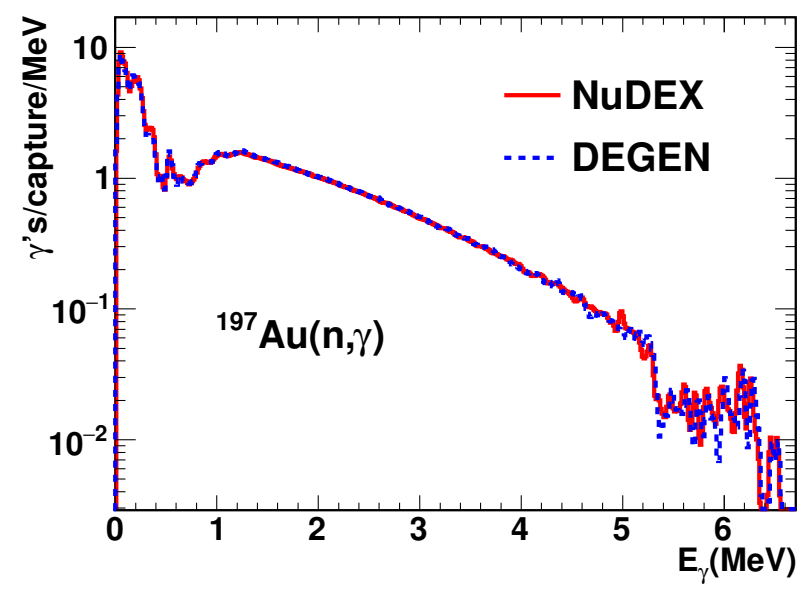

Figure 3. $\gamma$-ray spectra emitted after thermal ${ }^{197} \mathrm{Au}(\mathrm{n}, \gamma)$ reactions, calculated with NuDEX and DEGEN. The $\gamma$-ray spectra have been broadened by with a resolution $R=50 \mathrm{keV} / \mathrm{E}$.

present in the ENDF-6 format data libraries. On the contrary, G4PhotonEvaporation produces cascades which, although they conserve energy, are too simple at least in this case and which do not seem to correspond with reality.

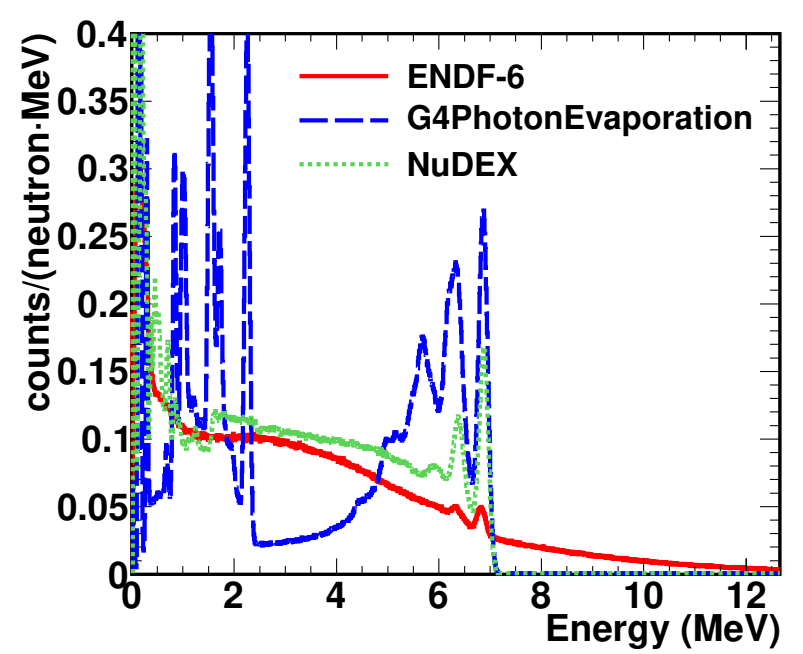

Figure 4. Response function to thermal neutrons simulated with Geant 4 of a CsI detector of $10 \mathrm{~cm}$ length and $10 \mathrm{~cm}$ in diameter. Three different capture $\gamma$-ray generators have been used: the default G4ParticleHP model, based on ENDF-6 data (ENDF-6), the G4PhotonEvaporation model, and NuDEX.

\section{Conclusions}

$\mathrm{NuDEX}$ is a recently developed code capable of generating correlated de-excitation $\gamma$-ray cascades, using the information present in the RIPL-3 and ENSDF nuclear structure data libraries. In this document we have briefly described how the code works. We have also presented some verification benchmarks, showing how NuDEX is able to reproduce the values from the CapGam database and the

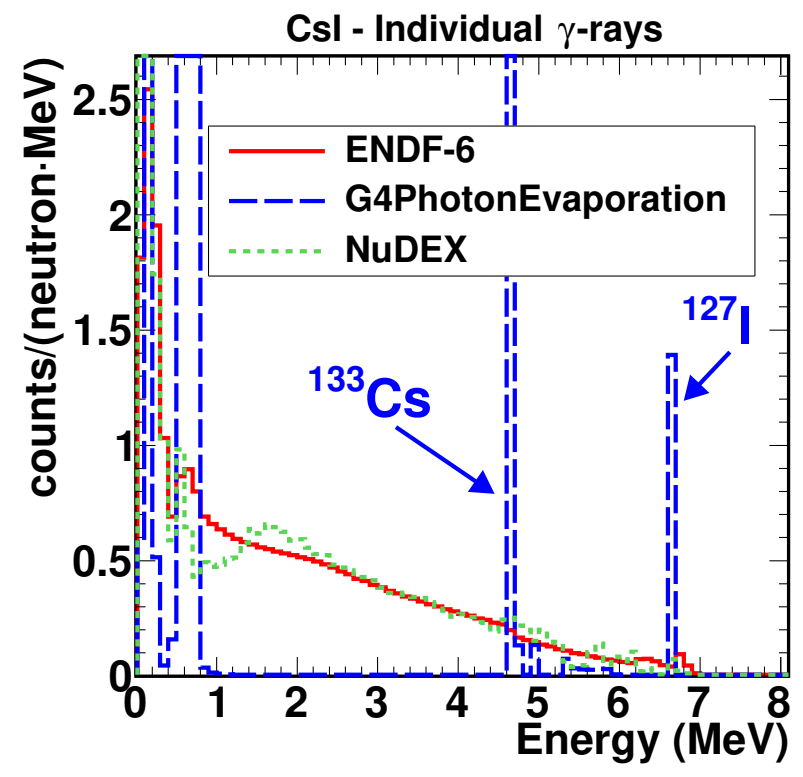

Figure 5. $\gamma$-ray energy spectra generated by Geant 4 in the simulations presented in Figure 4.

results from DEGEN. Finally, we have used it in the calculation of the response function of a CsI detector to thermal neutrons, and compared with other cascade generation models available in Geant4.

Acknowledgements: This work was supported in part by the Spanish national company for radioactive waste management ENRESA, through the CIEMAT-ENRESA agreements on "Transmutación de radionucleidos de vida larga como soporte a la gestión de residuos radioactivos de alta actividad"; by the Spanish Plan Nacional de I+D+i de Física de Partículas, through the projects FPA2014-52823C2-1-P, FPA2017-82647-P, FPA2017-83946-C2-1-P and PGC2018-096717-B-C21; and by the European Commission with the FP7 project CHANDA (FP7-605203).

\section{References}

[1] S. Agostinelli et al. (the GEANT4 Collaboration), Nucl. Instrum. Methods A 506, 250 (2003)

[2] T.G. others, Nuclear Technology 180, 298 (2012), https://doi .org/10.13182/NT11-135

[3] T. Böhlen, F. Cerutti, M. Chin, A. Fassò, A. Ferrari, P. Ortega, A. Mairani, P. Sala, G. Smirnov, V. Vlachoudis, Nucl. Data Sheets 120, 211 (2014)

[4] A. Trkov et al., Tech. Rep. BNL-203218-2018-INRE (2018)

[5] The JEFF team, JEFF-3.3: Evaluated nuclear data library (2017), https://www.oecd-nea. org/dbdata/jeff/jeff33/

[6] D. Brown et al., Nuclear Data Sheets 148, 1 (2018), special Issue on Nuclear Reaction Data

[7] K. Shibata, O. Iwamoto, T. Nakagawa, N. Iwamoto, A. Ichihara, S. Kunieda, S. Chiba, K. Furutaka, 
N. Otuka, T. Ohsawa et al., J. Nucl. Sci. Technol. 48, 1 (2011)

[8] F. Bečváŕ, Nucl. Instrum. Methods A 417, 434 (1998)

[9] D. Jordan, A. Algora, J. Tain, Nucl. Instrum. Methods A 828, 52 (2016)

[10] https://www.nndc.bnl.gov/ensdf/
[11] R. Capote et al., Nuclear Data Sheets 110, 3107 (2009)

[12] I. Band et al., Atomic Data and Nuclear Data Tables 81, 1 (2002)

[13] https://www.nndc.bnl.gov/capgam/

[14] J. Tain, D. Cano-Ott, Nucl. Instrum. Methods A 571, 719 (2007) 